\title{
The American Association for Thoracic Surgery Graham Traveling Fellowship, 2003-2004
}

\section{STIPEND $\$ 75,000$}

$T^{\text {he }}$ he American Association for Thoracic Surgery Evarts A. Graham Memorial Traveling Fellowship was established in 1951 by The American Association for Thoracic Surgery. Administered through the Graham Educational Research Foundation Fellowship grants support study by young cardiothoracic surgeons from outside North America at sites of their choice within North America and include travel between sites. Goals of the program are to broaden overall training and increase international contacts. Each Fellow should plan to have one primary center for his/her activities where he/she should spend from six to nine months. The Fellow should have identified an individual at that center who will act as his/her sponsor and assist in planning the course of study at the host institution and at other institutions. In addition to the primary center, the Fellow is encouraged to plan to spend shorter periods of time at other secondary centers to enhance the Fellowship experience.

Awards are made to surgeons of unique promise who have been regarded as having the potential for later international thoracic surgical leadership. Since the inception of the Graham Fellowship, 49 young surgeons from 26 countries have completed the Fellowship.

The Fellowship provides a stipend of $\$ 75,000$, a major portion of which is intended for traveling expenses incurred when visiting other medical centers. The Fellowship also provides two round-trip coach air fares from the recipient's country to his/her primary center. The dates of the beginning and ending of the Fellowship year should be scheduled to coincide with the Annual Meeting of The American Association for Thoracic Surgery in May 2003. The Fellow will be introduced at this meeting and his/her predecessor will give a report of his/her year's experience to the Plenary
Session. The Fellow will be expected to present a summary of his/her year's experience at the 2004 Annual Meeting, which will take place at the Metro Toronto Convention Center, Toronto, Ontario, Canada, April 25-28, 2004.

The Fellow should become involved predominantly with observation, consultation, teaching, and research at a variety of thoracic surgical training centers during the twelve months. Patient contact will be determined by the sponsoring surgeon and will conform to the regulations and licensing requirements of the state, province, or country in which he/she is studying. A candidate should have completed his/her formal training in general surgery and in thoracic and cardiovascular surgery, but he/she should not have reached a senior position. Candidates must be sufficiently proficient in English to realize the full benefits of the Fellowship. Candidates should not have received significant training in North America prior to making application for this award.

Applications for the Fellowship are available from:

Tirone E. David, MD, President

Graham Education and Research Foundation

Thirteen Elm Street

Manchester, MA 01944-1314

USA

Telephone 978-526-8330; fax 978-526-4018

Applications may be downloaded from the AATS Web site at: http://www.aats.org/doc/4939.

Completed applications must be returned no later than July 1, 2002. The selected candidate will be notified by December 15, 2002.

\section{Evarts A. Graham Memorial Traveling Fellows}

\begin{tabular}{|c|c|c|c|c|c|}
\hline lst & $1951-52$ & $\begin{array}{l}\text { L. L. Whytehead, MD, FRCS } \\
\text { Winnipeg, Manitoba, Canada }\end{array}$ & 4th & $1955-56$ & $\begin{array}{l}\text { Raymond L. Hurt, FRCS } \\
\text { Radlett Herts, England }\end{array}$ \\
\hline 2nd & $1953-54$ & $\begin{array}{l}\text { W. B. Ferguson, MB, FRCS } \\
\text { Newcastle-upon-Tyne, }\end{array}$ & 5 th & $1956-57$ & $\begin{array}{l}\text { Mathias Paneth, FRCS } \\
\text { London, England }\end{array}$ \\
\hline $3 \mathrm{rd}$ & $1954-55$ & $\begin{array}{l}\text { England } \\
\text { Lance L. Bromley, MChir, } \\
\text { FRCS } \\
\text { London, England }\end{array}$ & 7th & $1957-58$ & $\begin{array}{l}\text { Peter L. Brunnen, FRCS } \\
\text { Aberdeen, Scotland } \\
\text { N. G. Meyne, MD } \\
\text { Amsterdam, The Netherlands }\end{array}$ \\
\hline
\end{tabular}

The Journal of Thoracic and Cardiovascular Surgery • Volume 123, Number 3609 


\begin{tabular}{|c|c|c|c|c|c|}
\hline 8 th & $1960-61$ & $\begin{array}{l}\text { Godrej S. Karai, MD } \\
\text { Calcutta, India }\end{array}$ & 30th & $1980-81$ & $\begin{array}{l}\text { Richard Firmin, MD } \\
\text { London, England }\end{array}$ \\
\hline \multirow[t]{2}{*}{ 9th } & $1961-62$ & Fritz Helmer, MD & $31 \mathrm{st}$ & $1981-82$ & Claudio A. Salles, MD \\
\hline & & Vienna, Austria & & & Belo Horizonte, Brazil \\
\hline \multirow[t]{2}{*}{10 th } & $1962-63$ & Theodor M. Scheinin, MD & 32 nd & $1982-83$ & Yasuhisa Shimazaki, MD \\
\hline & & Oulu, Finland & & & Osaka, Japan \\
\hline \multirow[t]{2}{*}{ llth } & $1963-64$ & Masahiro Saigusa, MD & 33rd & $1983-84$ & Georg S. Kobinia, MD \\
\hline & & Tokyo, Japan & & & Linz, Austria \\
\hline \multirow[t]{2}{*}{12 th } & $1963-64$ & Adar J. Hallen, MD & 34 th & $1984-85$ & Aram Smolinsky, MD \\
\hline & & Uppsala, Sweden & & & Tel Hashomer, Israel \\
\hline \multirow[t]{2}{*}{13 th } & $1964-65$ & Stuart C. Lennox, MD & 35 th & $1985-86$ & Florentino J. Vargas, MD \\
\hline & & London, England & & & Buenos Aires, Argentina \\
\hline \multirow[t]{2}{*}{14 th } & $1964-65$ & Elias Carapistolis, MD, FACS & 36th & $1986-87$ & Ari L. J. Harjula, MD \\
\hline & & Thessaloniki, Greece & & & Helsinki, Finland \\
\hline \multirow[t]{2}{*}{15 th } & $1965-66$ & Gerhard Friehs, MD & 37 th & $1987-88$ & Byung-Chul Chang, MD \\
\hline & & Graz, Austria & & & Seoul, Korea \\
\hline \multirow[t]{2}{*}{16 th } & $1965-66$ & Ary Blesovsky, MD & 38th & $1988-89$ & Wang Cheng, MD \\
\hline & & London, England & & & Wuhan, The People's Republic \\
\hline \multirow[t]{2}{*}{17 th } & $1966-67$ & C. Peter Clarke, FRACS & & & of China \\
\hline & & Fitzroy, Australia & 39 th & $1989-90$ & Christopher J. Knott-Craig, MD \\
\hline \multirow[t]{2}{*}{18 th } & $1966-67$ & G. B. Parulkar, MD & & & Cape Town, Republic of South \\
\hline & & Bombay, India & & & Africa \\
\hline \multirow[t]{2}{*}{ 19th } & $1967-68$ & Claus Jessen, MD & 40th & 1991-92 & Ko Bando, MD \\
\hline & & Copenhagen, Denmark & & & Okayama, Japan \\
\hline \multirow[t]{2}{*}{ 20th } & $1969-70$ & Peter Bruecke, MD & 41 st & $1992-93$ & Timothy E. Oaks, MD \\
\hline & & Linz-Puchenau, Austria & & & Hershey, Pennsylvania, USA \\
\hline \multirow[t]{2}{*}{$21 \mathrm{st}$} & $1970-71$ & Michel S. Slim, MD & 42 nd & 1993-94 & Alain E. Serraf, MD \\
\hline & & Beirut, Lebanon & & & Creteil, France \\
\hline \multirow[t]{2}{*}{$22 \mathrm{nd}$} & $1971-72$ & Severi Pellervo Mattila, MD & $43 \mathrm{rd}$ & $1995-96$ & Cornelius McKown Dyke, MD \\
\hline & & Helsinki, Finland & & & Richmond, Va, USA \\
\hline \multirow[t]{2}{*}{$23 r d$} & $1972-73$ & Yasuyuki Fujiwara, MD & 44th & $1996-97$ & Monica Robotin-Johnson, MD \\
\hline & & Tokyo, Japan & & & Sydney, Australia \\
\hline \multirow[t]{2}{*}{ 24th } & $1973-74$ & Marc Roger de Leval, MD & 45 th & $1997-98$ & Jun Wang, MD \\
\hline & & London, England & & & Beijing, China \\
\hline \multirow[t]{3}{*}{25 th } & $1974-75$ & J. J. DeWet Lubbe, MD & 46th & 1998-99 & Christian Kreutzer, MD \\
\hline & & Bellville, Republic of South & & & Buenos Aires, Argentina \\
\hline & & Africa & 47 th & $1999-2000$ & Anders Franco-Cereceda, MD \\
\hline \multirow[t]{2}{*}{ 26th } & $1975-76$ & Mieczyslaw Trenkner, MD & & & Stockholm, Sweden \\
\hline & & Gdansk, Poland & 48th & $2000-01$ & Albertus Scheule, MD \\
\hline \multirow[t]{2}{*}{27 th } & 1976-77 & Bum Koo Cho, MD & & & Tuebingen, Germany \\
\hline & & Seoul, Korea & 49th & $2001-02$ & Anna Maria Ciccone, MD \\
\hline \multirow[t]{3}{*}{ 28th } & $1977-78$ & Alan William Gale, MD, & & & Rome, Italy \\
\hline & & FRACP, FRACS & 50 th & $2002-03$ & Cliff K. C. Choong, MD \\
\hline & & Sydney, Australia & & & Auckland, New Zealand \\
\hline \multirow[t]{2}{*}{ 29th } & $1978-79$ & Eduardo Otero Coto, MD & & & \\
\hline & & Valencia, Spain & & & \\
\hline
\end{tabular}

